\title{
Directivity of acoustic emissions from wave packets to the far field
}

\section{Journal Article}

\section{Author(s):}

Obrist, Dominik

Publication date:

2009-12

\section{Permanent link:}

https://doi.org/10.3929/ethz-b-000156719

Rights / license:

In Copyright - Non-Commercial Use Permitted

Originally published in:

Journal of Fluid Mechanics 640, https://doi.org/10.1017/S0022112009991297 


\title{
Directivity of acoustic emissions from wave packets to the far field
}

\author{
DOMINIK OBRIST $\dagger$ \\ Institute of Fluid Dynamics, ETH Zurich, 8092 Zurich, Switzerland
}

(Received 27 April 2009; revised 21 July 2009; accepted 21 July 2009; first published online

10 November 2009)

We investigate the acoustic emission from wave packets to the far field. To this end, we develop a theory for one- and two-dimensional source fields in the shape of wave packets with Gaussian envelopes. This theory is based on an approximation to Lighthill's acoustic analogy for distant observers. It is formulated in the spectral domain in which a Gaussian wave packet is represented again by a Gaussian. This allows us to determine the directivity of the acoustic emission (e.g. superdirectivity and Mach waves) by simple geometric constructions in the spectral domain. It is shown that the character of the acoustic emission is mainly governed by the aspect ratio and the Mach number of the wave packet source. To illustrate the relevance of this theory we use it to study two prominent problems in subsonic jet aeroacoustics.

\section{Introduction}

In order to understand the character of the acoustic emission from a given source, it is necessary to look at the temporal as well as the spatial structure of the source. The present work addresses sound sources shaped as wave packets. We will demonstrate the tight relation between the temporal and spatial structures of a wave packet source and its acoustic emission to the far field. In particular, we will study the relation between the aspect ratio and phase velocity of the wave packet and the directivity of the sound emission.

Wave packets have been identified by several authors as relevant structures in aeroacoustic source fields. Tam \& Morris (1980) discussed the sound emission from instability waves in shear layers. They showed that the spatial variation of the base flow leads to sound-emitting waves which are local in physical space and in spectral space (i.e. wave packets). This was followed by a study of the sound emission from wave packets in highly supersonic flows (Tam \& Burton 1984a,b). Laufer \& Yen (1983) performed experiments with low-Mach-number jets. They observed near-field pressure fluctuations in the shape of wave packets which exhibit highly directional acoustic emission patterns in the downstream direction. Such emission patterns are known as 'superdirective' acoustic fields (Crighton \& Huerre 1990) which cannot be expanded into finite series of multipoles. Their directional intensity is described by an exponential function of the emission angle which is called 'antenna factor' (Huerre \& Crighton 1983). Huerre \& Crighton (1983) and Crighton \& Huerre (1990) studied the superdirective sound emission from wave packet pressure fields analytically and tried to explain the observations of Laufer \& Yen (1983). Their wave packets feature different envelope functions in the axial direction, while they are compact in the

$\dagger$ Email address for correspondence: obrist@ifd.mavt.ethz.ch 
direction perpendicular to the jet axis. Haj-Hariri \& Akylas (1986) studied the weakly nonlinear temporal evolution of the acoustic emission from instability wave packets in boundary layers. They pointed out the sensitivity of the results to the shape of the wave packet envelope. Avital \& Sandham (1997) extended the results of Crighton \& Huerre (1990) and discussed the sound emitted by wave packet sources in the near and far fields for different Mach numbers and wave packet envelopes. Cooper \& Crighton (2000) performed a global stability analysis on a slowly diverging axisymmetric jet and found a global mode with a Gaussian envelope which produces a superdirective emission pattern. Up to small quantitative differences, their results were consistent with the experimental findings of Laufer \& Yen (1983). Wu (2005) derived an explicit solution for the Mach wave field due to a supersonic wave packet in terms of its envelope. Sandham, Morfey \& Hu (2006) investigated the aeroacoustics of a plane jet and found flow instabilities in the shape of two-dimensional wave packets which they then related to the sound generation. Le Dizès \& Millet (2007) studied the acoustic near field around instability wave packets for transonic configurations. They showed how the character of the acoustic emission changes as the waves in the source field pass from sub- to supersonic phase speed. Wu \& Huerre (in press) analysed the sound emitted by subsonic wave packets consisting of a pair of helical instability modes.

It is the goal of the present work to find an explanation for the different fundamental directivity patterns that can be observed for wave packet sources. But rather than trying to obtain a full solution for the complete acoustic field we will aim for an elementary theory which describes only the dominant terms in the acoustic far field. This will allow us to identify the relevant physical parameters characterizing the acoustic emission. To validate the theoretical predictions we will compare them to numerical results obtained with a standard acoustic solver. It integrates the retarded potential (derived from Lighthill's acoustic analogy) numerically in time for a known source field.

For the purpose of the present investigation, we focus on wave packets with a Gaussian envelope. Such wave packets have the convenient property that they can be represented by a Gaussian in the spectral domain. We will describe different emission patterns (superdirectivity, Mach waves, etc.) with a single theory which is based on a spectral formulation of Lighthill's acoustic analogy introduced in the following section. This formulation was also used by Bastin, Lafon \& Candel (1997) and Lesshafft (2006) to study the noise emission from jets. For simplicity, we will develop first a theory for a one-dimensional generic source in the shape of a Gaussian wave packet $(\S 2)$. In $\S 3$, we extend the theory to two-dimensional sources, where we will see that the aspect ratio of the wave packet has a major influence on the directivity pattern. As a further generalization, we look at oblique wave packets in $\S 4$, where we will find that Mach-wave-like sound emission is also possible for subsonic wave packets. In $\S 5$, we try to relate our theory to physical quantities in the source field. As an application of our theory, we offer in $\S 6$ some explanations for the sound emission from subsonic jets. Section 7 concludes this paper.

\subsection{Lighthill's acoustic analogy and the Fraunhofer approximation}

Lighthill's acoustic analogy (Lighthill 1952) relates the density $\rho$ to Lighthill's stress tensor $\boldsymbol{T}_{i j}$,

$$
\frac{\partial^{2} \rho}{\partial t^{2}}-a_{0}^{2} \frac{\partial^{2} \rho}{\partial x_{i} \partial x_{i}}=\frac{\partial^{2} \boldsymbol{T}_{i j}}{\partial x_{i} \partial x_{j}}
$$


with

$$
\boldsymbol{T}_{i j}=\rho u_{i} u_{j}+\left(p-a_{0}^{2} \rho\right) \delta_{i j}-\mu\left[\frac{\partial u_{i}}{\partial x_{j}}+\frac{\partial u_{j}}{\partial x_{i}}-\frac{2}{3} \frac{\partial u_{k}}{\partial x_{k}} \delta_{i j}\right],
$$

where $t$ is the time; $\boldsymbol{x}=\left(x_{1}, x_{2}, x_{3}\right)$ are the Cartesian coordinates; $u_{i}$ are the components of the velocity vector; $p$ is the pressure; $a_{0}$ is the ambient speed of sound; and $\mu$ is the dynamic viscosity. (Here and throughout this section we use Einstein's summation convention.)

If $\boldsymbol{T}_{i j}$ is known, (1.1) can be readily solved with the appropriate Green's function. In unbounded domains the pressure $p$ (which is equal to $a_{0}^{2} \rho$ in the isentropic far field) is then given by

$$
p(\boldsymbol{x}, t)=p_{\infty}+\left.\iiint_{-\infty}^{\infty} \frac{1}{4 \pi r} \frac{\partial^{2} \boldsymbol{T}_{i j}}{\partial x_{i} \partial x_{j}}\right|_{t-r / a_{0}} \mathrm{~d} \boldsymbol{x}^{\prime},
$$

where $p_{\infty}$ is the ambient pressure and $r=\left|\boldsymbol{x}-\boldsymbol{x}^{\prime}\right|$. The term $\partial^{2} \boldsymbol{T}_{i j} / \partial x_{i} \partial x_{j}$ is evaluated at the time $t-r / a_{0}$. This form is also known as the retarded potential. In the following sections, we will evaluate this integral numerically to validate our theoretical results.

To derive the theory for wave packets, we modify (1.3) for distant observers, i.e. $r_{o b s} \gg L$, where $L$ is a typical length scale of the source region. (We will give a sharper condition for $r_{o b s}$ later.) Further, we assume that $p$ and $\boldsymbol{T}_{i j}$ are periodic in time such that we may express these quantities as Fourier series,

$$
\begin{gathered}
p(\boldsymbol{x}, t)=\sum_{n=-\infty}^{\infty} \tilde{p}_{n}(\boldsymbol{x}) \mathrm{e}^{-\mathrm{i} \omega_{n} t}, \\
\frac{\partial^{2}}{\partial x_{i} \partial x_{j}} \boldsymbol{T}_{i j}(\boldsymbol{x}, t) \equiv q(\boldsymbol{x}, t)=\sum_{n=-\infty}^{\infty} \tilde{q}_{n}(\boldsymbol{x}) \mathrm{e}^{-\mathrm{i} \omega_{n} t},
\end{gathered}
$$

where $\omega_{n}=n 2 \pi / \tau$ in which $\tau$ is the period corresponding to the lowest resolved frequency. With these two Fourier series we can rewrite (1.3) as

$$
\tilde{p}_{n}(\boldsymbol{x})=\iiint_{-\infty}^{\infty} \frac{\tilde{q}_{n}\left(\boldsymbol{x}^{\prime}\right)}{4 \pi r} \mathrm{e}^{\mathrm{i} \omega_{n} r / a_{0}} \mathrm{~d} \boldsymbol{x}^{\prime} .
$$

In this form, the retarded time $t-r / a_{0}$ in (1.3) has translated into to a phase shift by the angle $\omega_{n} r / a_{0}$.

Without loss of generality, we assume now that the coordinate system $\boldsymbol{x}$ is oriented such that the observer is located at $\boldsymbol{x}=\left(r_{o b s}, 0,0\right)$ and that the source field $q$ is centred at the origin of $\boldsymbol{x}$. Based on these assumptions we approximate $r$ in (1.5) by

$$
r \approx r_{o b s}-x_{1}^{\prime} \text {. }
$$

This allows us to pull the 'overall phase shift' $\exp \left(\mathrm{i} \omega_{n} r_{o b s} / a_{0}\right)$ out of the integral. Further, we replace $1 / r$ by $1 / r_{o b s}$ which introduces an amplitude error $O\left(L / r_{o b s}\right)$ but no phase error. We write

$$
\tilde{p}_{n}(\boldsymbol{x})=\frac{\mathrm{e}^{\mathrm{i} \omega_{n} r_{o b s} / a_{0}}}{4 \pi r_{o b s}} \iiint_{-\infty}^{\infty} \tilde{q}_{n}\left(\boldsymbol{x}^{\prime}\right) \mathrm{e}^{-\mathrm{i} \omega_{n} x_{1}^{\prime} / a_{0}} \mathrm{~d} \boldsymbol{x}^{\prime} .
$$

The approximation that we have used to obtain (1.7) from (1.5) is known as the 'Fraunhofer approximation' (Howe 2003). 
From the definition of the spatial Fourier transform of $\tilde{q}$,

$$
\hat{q}_{n}(\boldsymbol{k})=\iiint_{-\infty}^{\infty} \tilde{q}_{n}(\boldsymbol{x}) \mathrm{e}^{-\mathrm{i} \boldsymbol{k} \cdot \boldsymbol{x}} \mathrm{d} \boldsymbol{x},
$$

it becomes clear that the integral in (1.7) is equal to the spatial Fourier coefficient of $\tilde{q}$ for $\boldsymbol{k}=\boldsymbol{k}_{o b s}=\left(\omega_{n} / a_{0}, 0,0\right)$. Therefore, we get

$$
\tilde{p}_{n}(\boldsymbol{x})=\frac{1}{4 \pi r_{o b s}} \hat{q}_{n}\left(\boldsymbol{k}_{o b s}\right) \mathrm{e}^{\mathrm{i} \omega_{n} r_{o b s} / a_{0}} .
$$

In a final step, we replace $\hat{q}_{n}$ by the spatio-temporal Fourier transform of $\boldsymbol{T}_{i j}$ and make use of the property that $\partial / \partial x_{i}$ translates to $\mathrm{i} k_{i}$,

$$
\tilde{p}_{n}(\boldsymbol{x})=-\frac{\omega_{n}^{2}}{4 \pi a_{0}^{2} r_{o b s}} \hat{\boldsymbol{T}}_{11, n}\left(\boldsymbol{k}_{\text {obs }}\right) \mathrm{e}^{\mathrm{i} \omega_{n} r_{o b s} / a_{0}} .
$$

For a general Cartesian coordinate system, the relation becomes

$$
\tilde{p}_{n}(\boldsymbol{x})=-\frac{k_{i} k_{j}}{4 \pi r_{o b s}} \hat{\boldsymbol{T}}_{i j, n}\left(\boldsymbol{k}_{\text {obs }}\right) \mathrm{e}^{\mathrm{i} \omega_{n} r_{o b s} / a_{0}},
$$

where

$$
\boldsymbol{k}_{\text {obs }}=\frac{\omega_{n}}{a_{0}} \frac{\boldsymbol{x}}{|\boldsymbol{x}|} .
$$

This relation tells us that only the energy contained in the wavenumber $\boldsymbol{k}_{\text {obs }}$ is emitted to the far field. All other Fourier components of the source field emit only into the near field. Or in other words, the acoustic wave guide is only able to transmit signals with the acoustic wavenumber $k_{a}=\omega_{n} / a_{0}$.

In one space dimension, sound of a given frequency $\omega$ can be emitted either in the positive $(x>0)$ or in the negative $(x<0)$ direction; i.e. there are only two wavenumbers, $k= \pm k_{a}$, which are relevant to the acoustic far field. In two dimensions, the relevant wavenumbers lie on a circle with radius $|\boldsymbol{k}|=k_{a}$. In three dimensions, the emitting wavenumbers lie on a sphere (cf. figure 1 of Goldstein 2005).

In the context of aeroacoustics (1.11) was probably first formulated by Kraichnan (1953). It was also derived in a slightly different context by Crighton (1975). Bastin et al. (1997) and Lesshafft (2006) used it to compute the sound in the far field of a plane jet and of a round hot jet, respectively. In this work, we will present a geometrical interpretation of (1.11) in the wavenumber plane.

So far, we have just stated that the Fraunhofer approximation is valid if the observer is far from the source region. We make this condition more precise by comparing the path lengths of two waves emitted from the edge and from the centre of the source region (figure 1). The Fraunhofer approximation requires that these two waves arrive at the observer with negligible phase difference, or in other words, the difference between the path lengths $r_{L}$ and $r_{o b s}$ must be much less than the acoustic wavelength $\lambda_{a}=2 \pi / k_{a}$,

$$
\sqrt{r_{o b s}^{2}+L^{2}}-r_{o b s} \ll \lambda_{a}
$$

For distant observers $\left(r_{o b s} \gg L\right)$ we can simplify this relation to obtain the following condition for the observer distance $r_{o b s}$ :

$$
\frac{r_{o b s}}{L} \gg \frac{L}{2 \lambda_{a}} .
$$




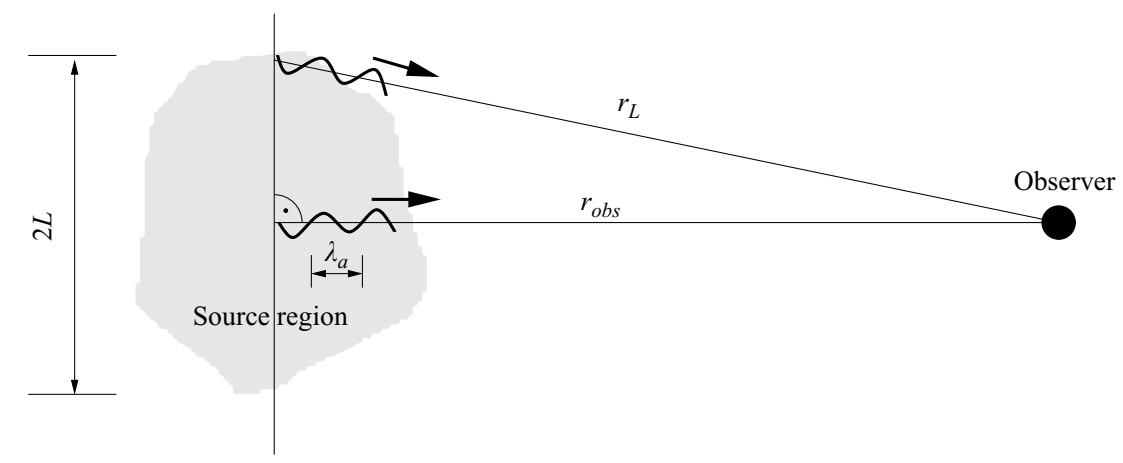

FIGURE 1. Different path lengths $r_{o b s}$ and $r_{L}$ for acoustic waves emitted from the centre and the edge of the source region, respectively.

In the following, we will define sources $q(\boldsymbol{x}, t)$ in the form of Gaussian wave packets. We postpone the discussion of the possible origin of such sources to $\S 7$.

\section{One-dimensional wave packets}

We begin with a one-dimensional problem for which we assume an acoustic source in the form of a wave packet of width $2 L$ :

$$
q(x, t)=q_{0} \mathrm{e}^{-x^{2} / L^{2}+\mathrm{i}\left(k_{0} x-\omega_{0} t\right)} .
$$

The waves within the wave packet envelope have a wavelength $\lambda_{0}=2 \pi / k_{0}$ and the phase velocity $c_{p}=\omega_{0} / k_{0}$. With this phase velocity, we define the Mach number $M_{p}$ as

$$
M_{p}=\left|\frac{c_{p}}{a_{0}}\right|=\left|\frac{k_{a}}{k_{0}}\right| .
$$

After a Fourier transform in time and space this source becomes

$$
\hat{q}(k, \omega)=q_{0} \mathrm{e}^{-\left(k-k_{0}\right)^{2} L^{2} / 4} \delta\left(\omega-\omega_{0}\right) .
$$

For simplicity, we will drop the functional dependence on $\omega$ in the following. According to the spectral formulation of the Fraunhofer approximation to Lighthill's analogy (1.10) only the wavenumber $k=k_{a}$ can emit sound to the far field. The energy contained in the other wavenumbers is irrelevant for the far field, except for the wavenumber $k=-k_{a}$ which corresponds to the sound emitted in the negative $x$-direction. This situation is illustrated in figure 2 .

The distance of the dominant wavenumber $k_{0}$ from the acoustic wavenumbers $\pm k_{a}$ determines the intensity of the emitted sound. Note that this is equally true for superand subsonic phase speed. The closer $k_{0}$ is to an acoustic wavenumber, the louder the emitted sound. If the distance of the dominant wavenumber to an acoustic line is less than $2 / L$ we speak of 'loud' wave packets; else we call them 'quiet' wave packets. Therefore, wave packets are loud (unidirectional) if

$$
\left|\frac{M_{p}}{M_{p}-1}\right|>\pi\left(\frac{L}{\lambda_{a}}\right),
$$

and they are loud in both directions (bidirectional) if

$$
\left|\frac{M_{p}}{M_{p}+1}\right|>\pi\left(\frac{L}{\lambda_{a}}\right) .
$$


(a)

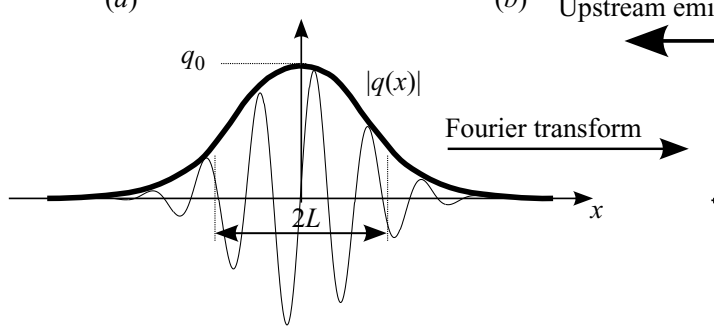

(b) Upstream emission

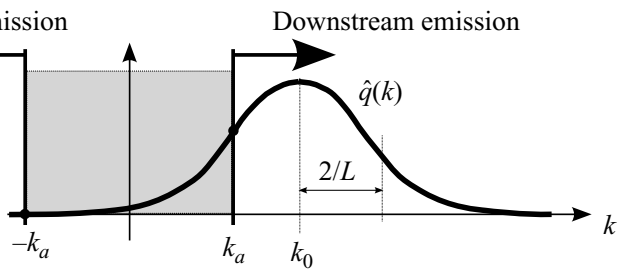

FIGURE 2. Representation of a one-dimensional wave packet in $(a)$ the physical and $(b)$ the spectral domain. The lines at $|k|=k_{a}$ indicate the wavenumbers which are relevant for the upand downstream emission of sound.

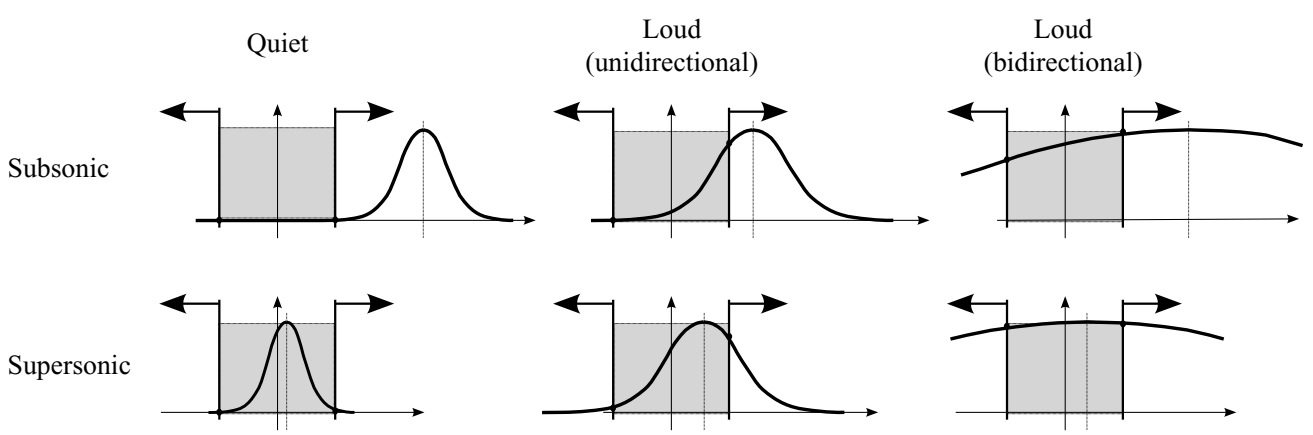

FigurE 3. Examples of quiet or loud wave packets with super- and subsonic phase speed.

Figure 3 illustrates six possibilities for quiet and (uni- and bidirectionally) loud sources with sub- and supersonic phase speed. It shows wave packets in the spectral domain (as in figure $2 b$ ). Their midpoint $k_{0}$ is either outside (subsonic) or inside (supersonic) the interval $\left[-k_{a}, k_{a}\right]$. The spectral width of the wave packets is proportional to the inverse of their physical width. The intensity of the emitted sound is indicated by the amplitude of the Gaussian as it intersects with the acoustic lines $\pm k_{a}$. Figure 3 shows also that the emission patterns do not differ fundamentally between wave packets with sub- or supersonic phase speed. It is only relevant whether the phase speed is close enough to the speed of sound. Fundamental differences between sub- and supersonic sound emission will only appear when we go to two-dimensional wave packets in $\S 3$.

According to (2.4) and (2.5), we can make a quiet wave packet loud by decreasing its width $L$. In the limit of $L \rightarrow 0$, the wave packets become monopole point sources which emit their energy equally in the up- and downstream directions. Of course, the distinction between loud and quiet sources is somewhat arbitrary, and one could argue that the right-hand sides of (2.4) and (2.5) should be multiplied by some other factor. In that sense, relations (2.4) and (2.5) should not be seen as strict quantitative rules. Rather, they indicate that the 'acoustic compactness' is the most relevant scale for the intensity of acoustic emission, where the acoustic compactness of a source is defined as the ratio of the size $L$ of the source to the acoustic wavelength $\lambda_{a}=\lambda_{0} / M_{p}$.

At this point, we would also like to emphasize that relations (2.4) and (2.5) do not indicate whether the sound emission is superdirective or not. We will show in the following sections that the intensity of the acoustic emission and the topology 


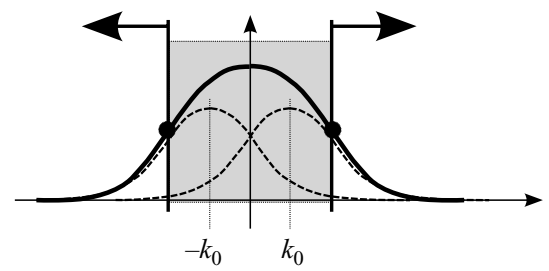

FIGURE 4. Sound emission from standing waves with wavenumber $k_{0}$.

(a)

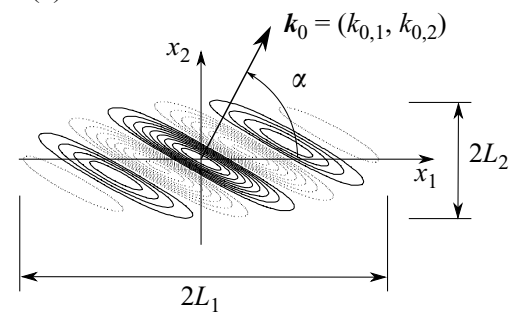

(b)

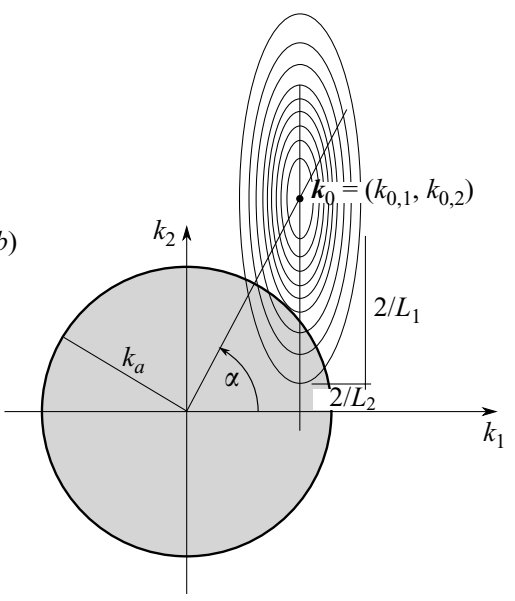

Figure 5. Contour lines (solid lines: positive values; broken lines: negative values) of a two-dimensional wave packet $\left(M_{p} \approx 0.7, \sigma \approx 3, \alpha=60^{\circ}\right)$ in $(a)$ the physical and $(b)$ the spectral domain. The shaded disk bounded by the acoustic circle $|\boldsymbol{k}|=k_{a}$ indicates supersonic wavenumbers.

of the directivity pattern are two different qualities which are influenced by different parameters.

Before we continue our discussion for two-dimensional wave packets, we want to discuss briefly the sound emission from wave packets with standing waves. We obtain standing waves by superimposing two wave packets with opposite wavenumbers $k_{0}$ and $-k_{0}$. Although such wave packets have no apparent phase velocity, we maintain the notion of the phase Mach number $M_{p}=k_{a} /\left|k_{0}\right|$. The sound emission from these wave packets is a superposition of the emission from the two symmetric wave packets (figure 4). The rule (2.4) for loud wave packets remains valid, except that the sound emission will always be bidirectional.

\section{Two-dimensional wave packets}

By going from one- to two-dimensional wave packet sources we introduce new length scales. We have now two wavenumbers $\left(k_{0,1}, k_{0,2}\right)$ and two widths $\left(L_{1}, L_{2}\right)$,

$$
q\left(x_{1}, x_{2}, t\right)=q_{0} \mathrm{e}^{-x_{1}^{2} / L_{1}^{2}-x_{2}^{2} / L_{2}^{2}+\mathrm{i}\left(k_{0,1} x_{1}+k_{0,2} x_{2}-\omega_{0} t\right)} .
$$

The slenderness of this wave packet is described by the aspect ratio $\sigma=L_{1} / L_{2}$. We will see in the following that $\sigma$ has a strong influence on the directivity of the sound emission. In the spectral domain, wave packet (3.1) is again a wave packet centred at $\left(k_{0,1}, k_{0,2}\right)$ with the half-widths $2 / L_{1}$ and $2 / L_{2}$ (figure 5 ). Therefore, the aspect ratio in the spectral domain is $1 / \sigma$. The waves inside the wave packet propagate in the 

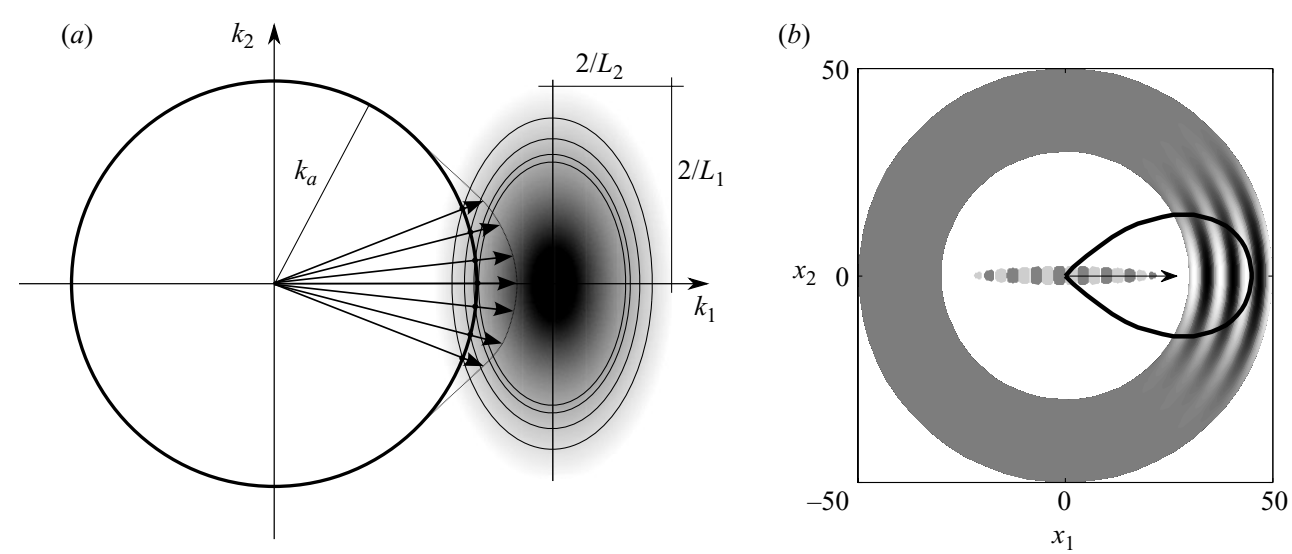

FIGURE 6. Superdirective sound emission to the far field from a slender subsonic wave packet. (a) The diagram shows how the acoustic circle $|\boldsymbol{k}|=k_{a}$ cuts through the spectral representation of the wave packet centred at $\left(k_{0}, 0\right)$. $(b)$ Numerical experiment with a slender subsonic wave packet $\left(\sigma=10, M_{p}=0.9\right)$. The shaded area in the centre illustrates the wave packet source field $q$ (bright: positive values; dark: negative values). The arrow indicates the propagation direction $\alpha$ of the waves within the wave packet. The thick line shows the SPL as a polar plot in which the radius corresponds to the SPL, and the polar angle indicates the emission direction $\varphi$. (Negative SPL values are not shown.) To illustrate the emitted acoustic waves the shaded ring surrounding the source shows a snapshot of $p\left(x_{1}, x_{2}, t_{0}\right) \cdot r$. The results were obtained by a direct numerical integration of Lighthill's analogy (1.3).

direction $\alpha=\arctan \left(k_{0,2} / k_{0,1}\right)$. Their phase velocity is given by $c_{p}=\omega /\left|\boldsymbol{k}_{0}\right|$ and the Mach number is

$$
M_{p}=\frac{k_{a}}{\left|\boldsymbol{k}_{0}\right|},
$$

where $\boldsymbol{k}_{0}=\left(k_{0,1}, k_{0,2}\right)$ is the wavenumber vector.

In the following, we use some simple geometric constructions in the spectral domain to illustrate and classify the different directivity patterns. We will begin our discussion for wavenumber vectors $\boldsymbol{k}_{0}$ which point in the streamwise direction, i.e. $k_{0,2}=0$ and $\alpha=0^{\circ}$. Wave packets with oblique waves $\left(\alpha \neq 0^{\circ}\right)$ will be discussed in $\S 4$.

\subsection{Subsonic phase velocity}

Let us start with a slender wave packet $(\sigma>1)$ with subsonic phase velocity and the wavenumber vector $\boldsymbol{k}_{0}=\left(k_{0}, 0\right)$. In the spectral domain, the centre of this wave packet lies outside the acoustic circle $|\boldsymbol{k}|=k_{a}$ because $k_{0}>k_{a}$ (figure $6 a$ ).

We know from (1.11) that only the Fourier modes $\hat{q}\left(|\boldsymbol{k}|=k_{a}\right)$ emit acoustic energy to the far field and that this energy is emitted in the direction of the wavenumber vector $\boldsymbol{k}$. This acoustic emission is illustrated in figure 6(a) by arrows. The direction of dominant sound emission is indicated by the tangential intersection of the spectral contours of the source field with the acoustic circle. In our case, the subsonic wave packet emits foremost in the downstream direction. The emission in the sideline and upstream directions is exponentially small. This directivity characteristic corresponds to the phenomenon of 'superdirective' emission (Crighton \& Huerre 1990).

We confirm our theoretical result by a numerical experiment. To this end, we evaluate integral (1.3) numerically for a given source term (3.1) with $\sigma=8$ and 

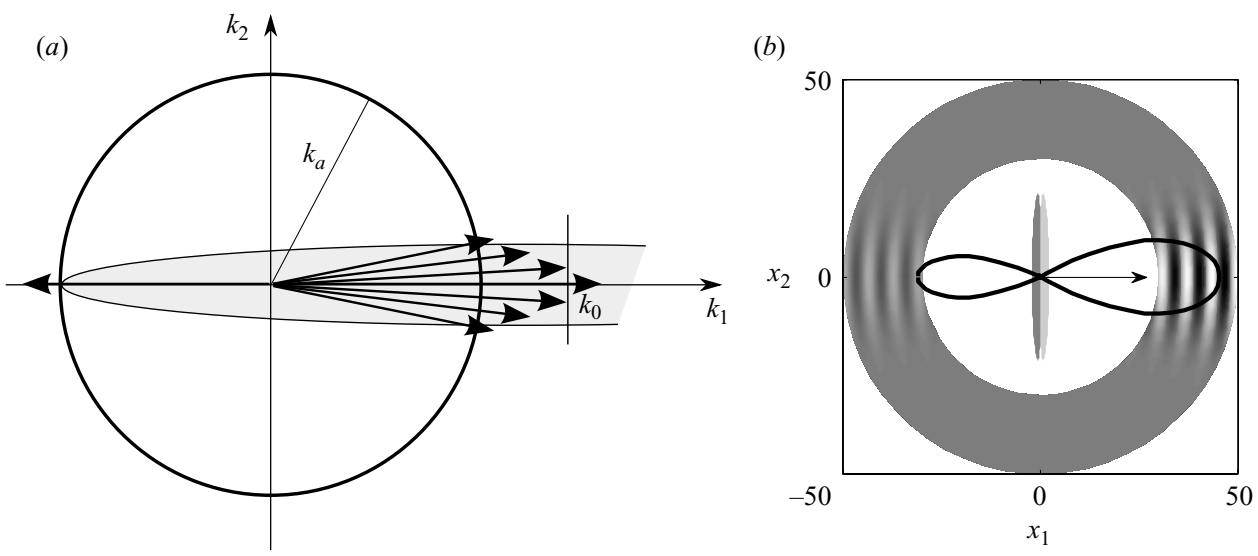

FigURE 7. Bidirective emission from a broad subsonic wave packet: $(a)$ diagram in the spectral domain; $(b)$ numerical experiment with $M_{p}=0.9$ and $\sigma=0.1$. (See the caption of figure $6 b$ for a more detailed explanation of $b$.)

$M_{p}=0.9$. We measure the intensity of the sound emission by the sound pressure level (SPL),

$$
\mathrm{SPL}=\log \left(\left|p^{\prime}\right| / p_{\text {ref }}\right)^{2},
$$

where the pressure fluctuation $p^{\prime}=p(\boldsymbol{x}, t)-p_{\infty}$ is, in general, a function of space and time. For our purpose, it is sufficient to measure the SPL along a large circle around the source field $q$. The SPL is then only a function of the emission angle $\varphi$ (measured from the $x_{1}$-axis). The reference pressure $p_{\text {ref }}$ is chosen arbitrarily to adjust the SPL values to a useful range. Figure $6(b)$ shows the result of this numerical experiment for a subsonic wave packet. It confirms our theoretical finding that almost all acoustic energy is emitted in the downstream direction. Because the numerical results are based on the full retarded potential (1.3) without any approximations, this result validates implicitly the applicability of the Fraunhofer approximation in the present context.

From figure 6(a) it becomes clear that all sufficiently slender subsonic wave packets are superdirective. For $\sigma<1$, however, we may obtain a directivity pattern which is reminiscent of a dipole (figure 7). We call this a 'bidirective' emission. At this point, it is important to emphasize the difference between the bidirective emission pattern and an actual dipole. While a dipole does not emit any sound at all in the sideline direction $\left(\varphi=90^{\circ}\right)$, the bidirective source emits an exponentially small amount of acoustic energy also in this direction.

To determine whether the emission pattern has superdirective or bidirective character, we have to look at the local radius of curvature of the ellipse centred at $\left(k_{0}, 0\right)$ which intersects tangentially with the acoustic circle at $\left(-k_{a}, 0\right)$ (figure $\left.7 a\right)$. It is straightforward to show that the radius of curvature of the ellipse at this point is $\sigma^{2}\left(\left|k_{0}\right|+k_{a}\right)$. If this radius is smaller than the radius of the acoustic circle, $k_{a}$, we have a bidirective emission pattern. Otherwise, the emission to the far field is superdirective. From this, we obtain the following condition for bidirective sound emission:

$$
\sigma<\sqrt{\frac{M_{p}}{M_{p}+1}} \quad \text { or } \quad M_{p}>\frac{\sigma^{2}}{1-\sigma^{2}} .
$$

This relation is illustrated and verified in figure 8. It shows the results of a numerical integration of (1.3) for a wave packet source with $\sigma=0.5$. As we increase stepwise 

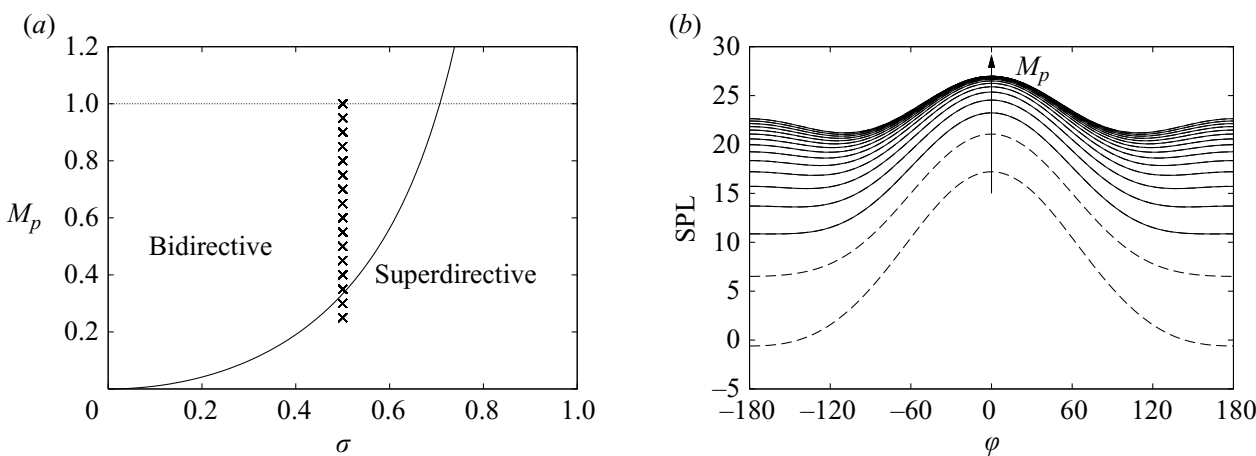

FIGURE 8. Transition from superdirective to bidirective emission for broad subsonic wave packets: The solid line in (a) illustrates the bidirectivity condition (3.4). The crosses in (a) indicate the parameter values for the numerical experiment shown in $(b)(\sigma=0.5$ and $\left.M_{p}=0.25,0.30, \ldots, 1\right)$. The solid lines in $(b)$ correspond to values of $M_{p}$ for which (3.4) is satisfied, whereas the broken lines indicate that the bidirectivity condition is not satisfied.

the Mach number of this source from 0.25 to 1 , we observe the transition form superdirective to bidirective emission as predicted by (3.4). For small Mach numbers, the SPL curves have a single local maximum at $\varphi=0^{\circ}$ (superdirectivity). As soon as condition (3.4) is satisfied the curves feature a second local maximum at $\varphi= \pm 180^{\circ}$ (bidirectivity).

The intensity of the sound emission in the down- and upstream directions is again determined by the loudness conditions (2.4) and (2.5), respectively. Here, we would like to stress again the difference between the emission pattern (super- or bidirective) and the loudness (uni- or bidirectional). The loudness criteria give us only information about the intensity of the emission in the up- and downstream directions, whereas the bidirectivity condition (3.4) indicates only whether the SPL has one (superdirective) local maximum or two (bidirective) local maxima. The most relevant parameter for the sound intensity is the acoustic compactness $L_{1} / \lambda_{a}$, while it is the aspect ratio $\sigma$ which determines the topology of the directivity pattern.

\subsection{Supersonic phase velocity}

If the phase speed of the waves within the wave packet envelope is supersonic we may obtain Mach waves. In the traditional theory of Mach waves the angle at which the Mach waves are emitted is given by $\vartheta_{c}=\arccos \left(1 / M_{p}\right)$ (Phillips 1960). This formula is the result of a simple geometric construction in the wavenumber plane for sinusoidal travelling waves. $\mathrm{Wu}$ (2005) derived formulas for the dominant emission direction and intensity for supersonic wave packets which are slowly modulated in the streamwise direction. Here, we discuss supersonic wave packets with Gaussian envelopes (figure 9). For very slender wave packets, $\sigma \gg 1$, the wave packet in the spectral domain cuts through the acoustic circle $|\boldsymbol{k}|=k_{a}$ at $\vartheta \approx \arccos \left(1 / M_{p}\right)$, and we obtain classical Mach waves. For wave packets with $\sigma \approx 1$, however, the inscribed ellipse with aspect ratio $1 / \sigma$ will intersect tangentially the acoustic circle at $\left(k_{a}, 0\right)$ such that the dominant emission angle is $\vartheta=0^{\circ}$ and the emission has superdirective character. The transition from this superdirective emission to Mach waves occurs when the curvature of the source field at the point $\left(k_{a}, 0\right)$ in the wave number plane becomes larger than the curvature of the acoustic circle. Therefore, Mach waves exist only if

$$
\sigma>\sqrt{\frac{M_{p}}{M_{p}-1}} \quad \text { or } \quad M_{p}>\frac{\sigma^{2}}{\sigma^{2}-1} .
$$



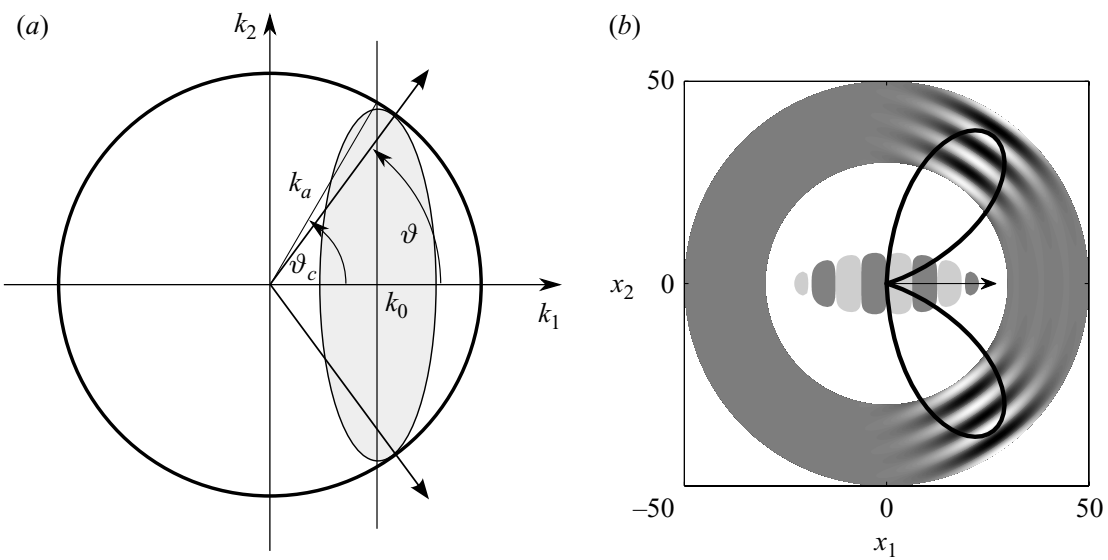

FiguRE 9. Emission of Mach waves a supersonic wave packet with $M_{p}=2$ and $\sigma=3$. (a) Diagram showing the construction of the dominant emission direction $\vartheta$ (different from the classical Mach angle $\cos \vartheta_{c}=1 / M_{p}$ ). (b) Numerical experiment. (See the caption of figure $6 b$ for a more detailed explanation of $b$.)
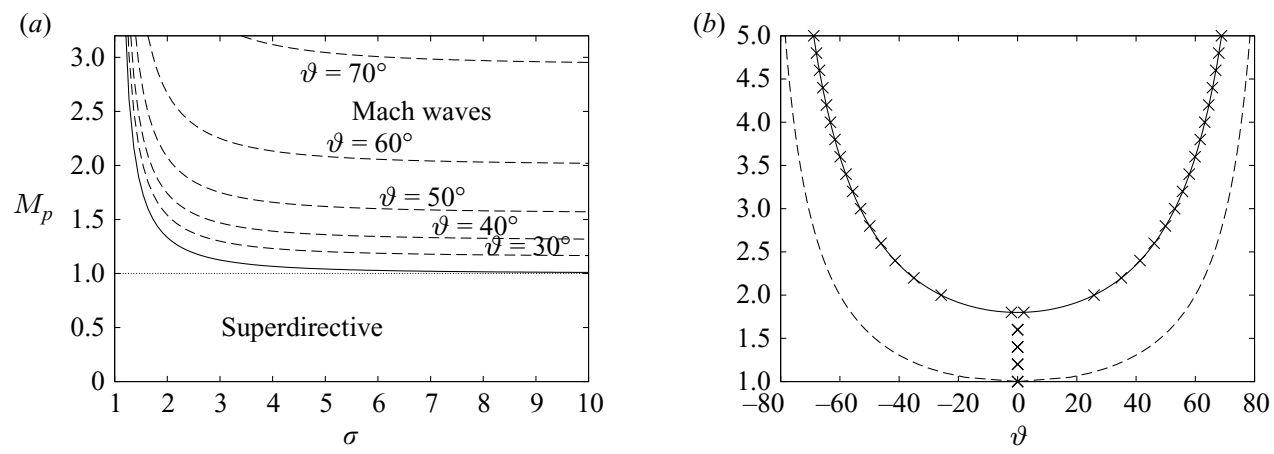

Figure 10. (a) Condition (3.5) for Mach waves; the broken lines indicate the corrected Mach angle (3.6). (b) Dominant emission direction as function of the Mach number $M_{p}$ for wave packets with aspect ratio $\sigma=1.5: \times$, numerical experiment; — (3.6); ---, classical Mach angle $\vartheta_{c}=\arccos 1 / M_{p}$.

This relation is visualized in figure $10(a)$ which shows that Mach waves are only possible for sufficiently slender and sufficiently fast wave packets. Furthermore, a numerical experiment (figure $10 b$ shows that the classical relation $\vartheta_{c}=\arccos \left(1 / M_{p}\right)$ over-predicts the dominant emission angle. The classical relation for the Mach angle is only valid for infinitely slender wave packets. For wave packets with finite aspect ratio we can derive the corrected Mach angle as

$$
\cos \vartheta=\frac{1}{M_{p}} \frac{\sigma^{2}}{\sigma^{2}-1} .
$$

Note that this expression is consistent with (3.5), as it assumes the limiting value of (3.5) if we set $\vartheta=0^{\circ}$. Furthermore, we can recover the formula for the classical Mach angle by letting $\sigma$ go to infinity.

Apart from the Mach waves (figure $9 b$ ) and the superdirective emission (figure 11b) we may also obtain a bidirective emission pattern (figure 11c). This is the case if the wave packet is so broad $(\sigma<1)$ that the ellipse which intersects tangentially at $\left(-k_{a}, 0\right)$ has a radius of curvature smaller than $k_{a}$ (figure 11a). It turns out that 
the condition for supersonic bidirectivity is equivalent to condition (3.4) for subsonic sources.

\subsection{Unified theory}

In the previous sections, we have derived several criteria for characterizing the directivity of sound emission for two-dimensional wave packet sources with $\alpha=0^{\circ}$. We observe that expressions (3.4)-(3.6) are formally very similar. As a matter of fact, we will show in the following that the results of the previous two sections can be unified in a single theory.

To this end, we find first a general solution for the sound emission from twodimensional wave packet sources. According to the spectral form of the Fraunhofer approximation of Lighthill's analogy (1.9), the Fourier transform of our wave packet source,

$$
\hat{q}\left(k_{1}, k_{2}\right)=q_{0} \mathrm{e}^{-\left(k_{1}-k_{0,1}\right)^{2} L_{1}^{2} / 4-\left(k_{2}-k_{0,2}\right)^{2} L_{2}^{2} / 4},
$$

can be used to determine the sound emitted to the far field. We can obtain an explicit expression for the sound emission by parameterizing (3.7) along the acoustic circle; i.e. we set $\left(k_{1}, k_{2}\right)=\left(k_{a} \cos \varphi, k_{a} \sin \varphi\right)$, where $\varphi$ is the emission angle measured from the $x_{1}$-axis. After some rearrangement of terms, we obtain the general solution for the sound emission from two-dimensional wave packet sources,

$$
\tilde{p}(\varphi) \sim \exp \left\{-\frac{\pi^{2} L_{1}^{2}}{M_{p}^{2} \lambda_{a}^{2}}\left[\left(\cos \alpha-M_{p} \cos \varphi\right)^{2}+\frac{1}{\sigma^{2}}\left(\sin \alpha-M_{p} \sin \varphi\right)^{2}\right]\right\} .
$$

For the special case in which the wave propagates in the $x_{1}$-direction $\left(\alpha=0^{\circ}\right)$ and in which the wave packet is compact in the $x_{2}$-direction $(\sigma \rightarrow \infty)$ we recover immediately the antenna factor by Huerre \& Crighton (1983). Note that the Fraunhofer approximation does not reproduce any multipole terms $\left(\cos ^{2} \varphi, \cos ^{4} \varphi\right.$, etc.). These contributions were discarded in $\S 1.1$ with the so-called amplitude error.

Based on the general solution (3.8) and the definition of the SPL (3.3) we can now find an expression for the difference $\triangle$ SPL in the SPL between down- and upstream emissions, i.e. $\varphi=0^{\circ}$ and $180^{\circ}$,

$$
\Delta \mathrm{SPL}=\frac{8 \pi^{2}}{M_{p}}\left(\frac{L}{\lambda_{a}}\right)^{2} .
$$

Similar to the loudness criteria (2.4) and (2.5), the difference in $\triangle$ SPL is mainly governed by the acoustic compactness of the source.

Next, we derive an analytical expression for the relative curvature $\kappa$ of $\operatorname{SPL}(\varphi)$ at $\varphi=0^{\circ}$. If we define $\kappa$ as

we find from (3.8) that

$$
\kappa \equiv-\left.\frac{2}{\Delta \operatorname{SPL}} \frac{\partial^{2}}{\partial \varphi^{2}} \operatorname{SPL}\right|_{\varphi=0^{\circ}}
$$

$$
\kappa=1-M_{p}+\frac{M_{p}}{\sigma^{2}} .
$$

Geometrically, the relative curvature $\kappa$ indicates whether the SPL curves (e.g. the curves in figure $8 b$ or the thick lines in figures $6 b, 7 b, 9 b, 11 b, 11 c$, etc.) have a local minimum $(\kappa<0)$ or maximum $(\kappa>0)$ at $\varphi=0^{\circ}$ (given that the sound emission is symmetric with respect to the $x_{1}$-axis). If we rewrite (3.11) as

$$
M_{p}=(1-\kappa) \frac{\sigma^{2}}{1-\sigma^{2}}
$$



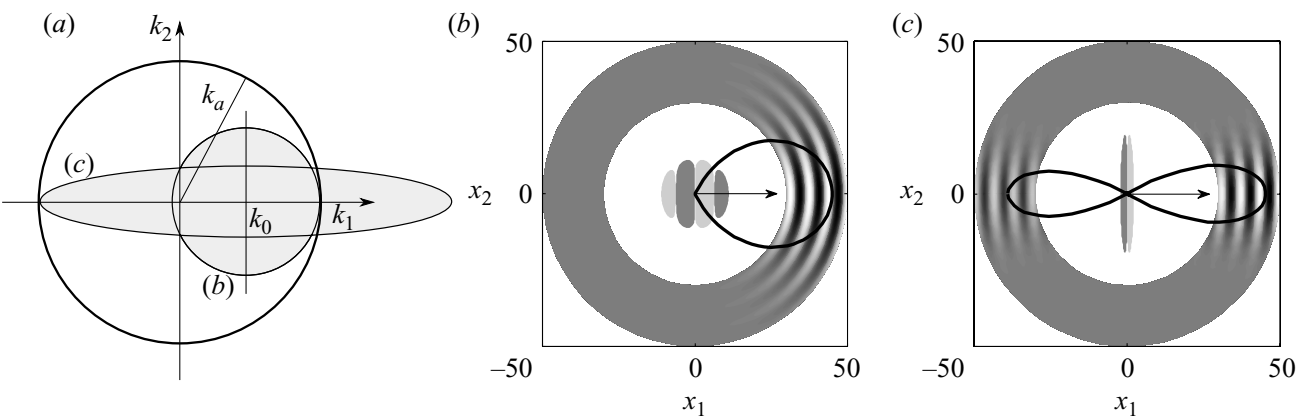

FIGURE 11. (a) Emission from supersonic wave packets without Mach waves: $(b)$ superdirectivity $\left(M_{p}=2, \sigma=1\right),(c)$ bi-directivity $\left(M_{p}=2, \sigma=0.1\right)$. (See caption of figure $6 b$ for a more detailed explanation of $b$ and $c$.)

we see immediately a connection to the conditions for bidirective wave packets (3.4) and for Mach waves (3.5). We can recover these relations by setting $\kappa=2$ and $\kappa=0$, respectively. Therefore, curvatures larger than 2 indicate bidirective emission. Positive curvatures smaller than 2 (i.e. $0<\kappa<2$ ) indicate superdirectivity, and negative curvatures indicate Mach waves. From (3.12) it becomes also clear that Mach waves, i.e. $\kappa<0$ are inherently tied to supersonic wave packets $\left(M_{p}>1\right)$. Further, a comparison of (3.12) with the expression for the corrected Mach angle (3.6) shows that there exists a direct relation between the corrected Mach angle $\vartheta$ and the (negative) curvature $\kappa$,

$$
\cos \vartheta=\frac{1}{1-\kappa} \quad \text { for } \kappa<0 .
$$

We summarize these results in figure 12. It shows contours of constant $\kappa$ as a function of the aspect ratio $\sigma$ and the Mach number $M_{p}$. The contour lines for $\kappa=0$ and $\kappa=2$ divide the $\left(\sigma, M_{p}\right)$-plane into three different domains: bidirective emission (cf. figures $7 b$ and 11c), superdirective emission (cf. figures $6 b$ and $11 b$ ) and Mach waves (cf. figure 9b). And as we have learned from (3.13), the contour lines in the Mach wave domain are also lines of constant dominant emission angle $\vartheta$ (cf. figure $10 a$ ).

\section{Oblique wave packets}

In the previous section, we have derived a theory for the directivity of the acoustic emission from two-dimensional wave packets with waves propagating along one of the principal axes of the wave packet $\left(\alpha=0^{\circ}\right)$. In this section, we relax this constraint and study wave packets with oblique wave propagation $\left(\alpha \neq 0^{\circ}\right)$. We will find that such sources exhibit more complex emission patterns.

As an example, figure 13 shows a numerical experiment with three oblique wave packet sources with subsonic phase velocity. All three wave packets have the same Mach number $M_{p}$ and the same wave propagation direction $\alpha$. They only differ in their aspect ratio $\sigma$. We observe that all emission patterns have a superdirective character and that the angle $\vartheta$ of the dominant emission direction is apparently a function of the aspect ratio $\sigma$. For large $\sigma$, subsonic oblique wave packets emit mostly into the downstream direction. As the aspect ratio $\sigma$ decreases, the acoustic emission shifts to higher emission angles $\vartheta$. For $\sigma=1$ the dominant emission angle $\vartheta$ coincides 


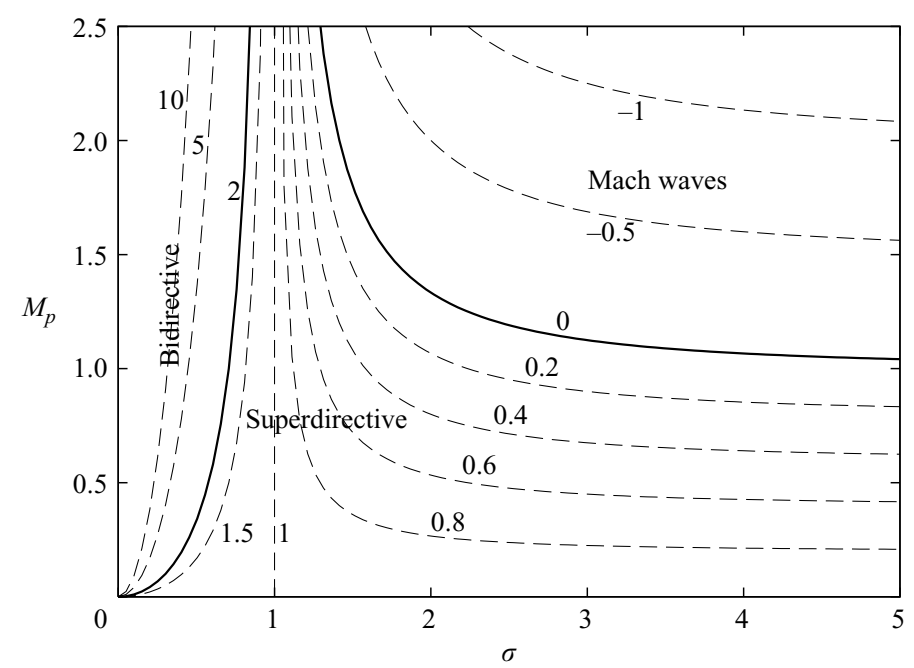

FIGURE 12. Composite diagram showing the domains of bidirective emission, superdirectivity and Mach waves as a function of the phase Mach number $M_{p}$ and the aspect ratio $\sigma$. The dashed lines with numbers indicate the values of the curvature $\kappa$. For negative curvatures the broken lines are identical with curves of constant Mach angle (cf. figure 10a).

(a)

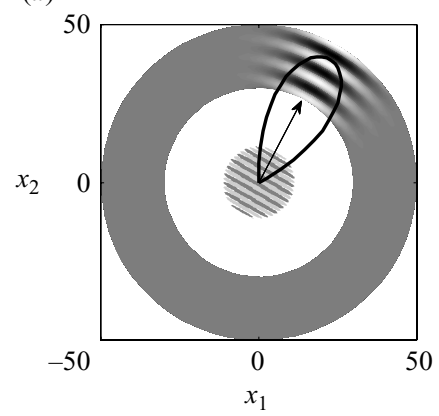

(b)

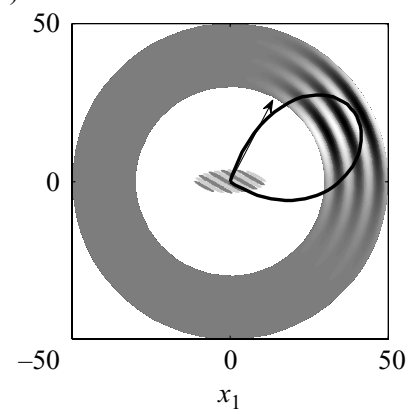

(c)

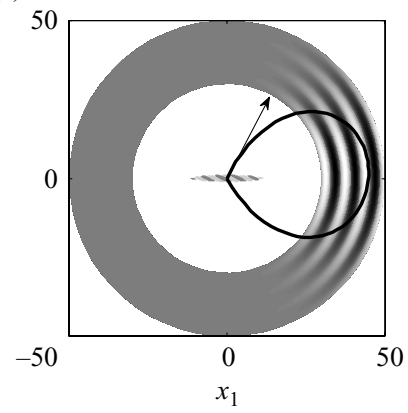

FIGURE 13. Oblique wave packets with $M_{p}=0.4, \alpha=60^{\circ}$ and $(a) \sigma=1,(b) \sigma=3,(c) \sigma=10$. (See the caption of figure $6 b$ for a more detailed explanation of these figures.)

exactly with the wave propagation angle $\alpha$ which is, of course, an obvious result if we consider the high level of symmetry of this configuration.

We can explain the observations for subsonic oblique wave packets by similar reasoning as in the previous section. In figure 14(a) we show that the dominant emission angle $\vartheta$ can be found by constructing an ellipse centred at $\left(k_{0,1}, k_{0,2}\right)$ with aspect ratio $1 / \sigma$ which intersects the acoustic circle tangentially. The point of tangential intersection indicates the dominant emission angle $\vartheta$. It can be shown that this angle satisfies the relation

$$
\sigma=\sqrt{\frac{\cos \vartheta\left(\sin \alpha-M_{p} \sin \vartheta\right)}{\sin \vartheta\left(\cos \alpha-M_{p} \cos \vartheta\right)}}
$$




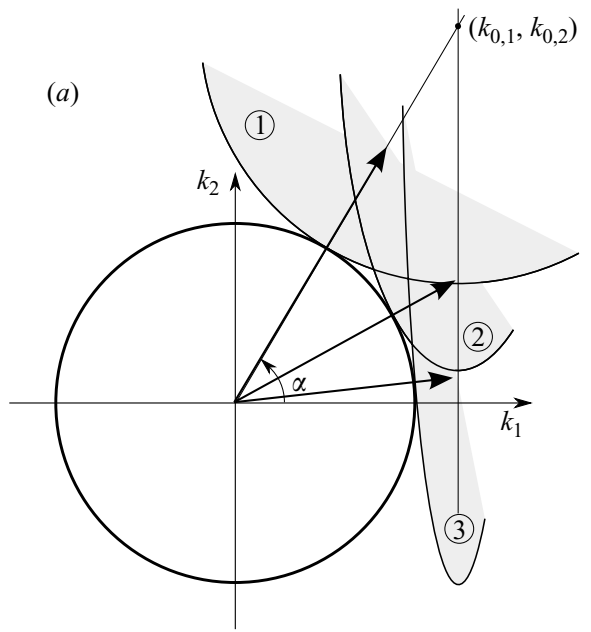

(b)

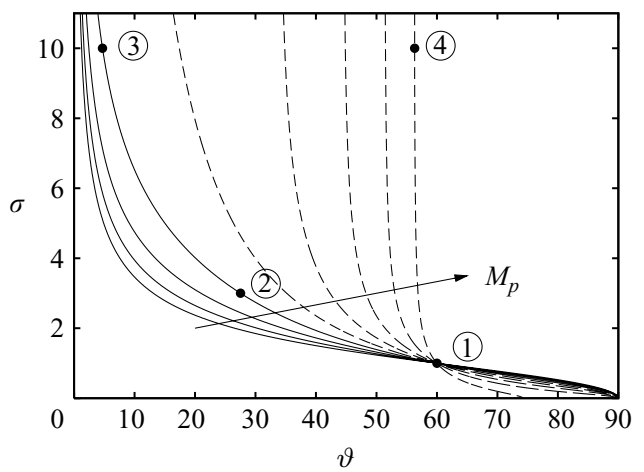

FigURE 14. (a) Directivity of sound emission from oblique subsonic wave packets with different aspect ratios $\left(M_{p}=0.4, \alpha=60^{\circ}\right)$. (b) Dominant angle of emission $\vartheta$ as a function of $\sigma$ for oblique wave packets with $\alpha=60^{\circ}$ and $M_{p}=0.1, \ldots, 0.9$. The broken lines indicate that one of the projected Mach numbers, $M_{p} / \cos \alpha$ or $M_{p} / \sin \alpha$, is supersonic. (The labels (1), (2) and (3) correspond to figure $13 a-c$, respectively. The label (4) refers to the wave packet shown in figure 15.)

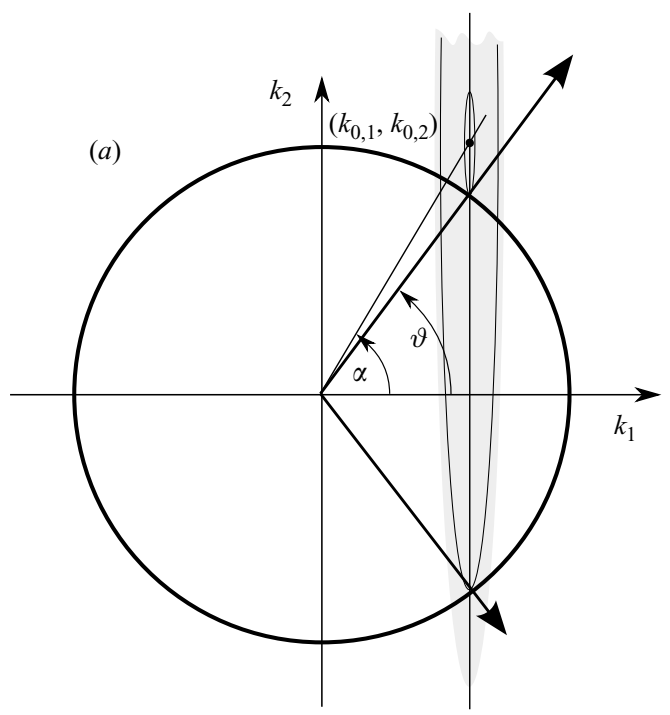

(b)

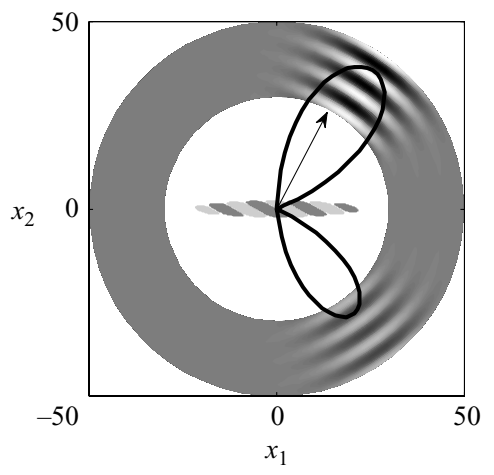

FIGURE 15. Mach-wave-like emission from an oblique wave packet with $M_{p}=0.9, \alpha=60^{\circ}$ and $\sigma=10$. (See the caption of figure $6 b$ for a more detailed explanation of $b$.)

Note that we can formally recover the expression for the corrected Mach angle (3.6) if we set $\alpha=0^{\circ}$.

In the case in which the projection of the Mach number on the $x_{1}$ - or $x_{2}$-axis $\left(M_{p} / \cos \alpha\right.$ or $M_{p} / \sin \alpha$, respectively) is supersonic, we may obtain a situation as shown in figure 15 which produces Mach-wave-like emission with two lobes. Unlike the real Mach waves from $\S 3.2$, this emission pattern is asymmetric such that the main lobe which is approximately aligned with the phase velocity of the source has a 
higher intensity (and a slightly higher emission angle) than the secondary lobe. Even in that case, relation (4.1) predicts the dominant emission angle (of the main lobe) correctly. However, either the nominator or the denominator of (4.1) will become zero for a finite angle $\vartheta$ such that the range of possible emission angles $\vartheta$ does not span the full range from $0^{\circ}$ to $90^{\circ}$.

Figure $14 b$ illustrates relation (4.1) for oblique wave packets with $\alpha=60^{\circ}$. We see that the dominant emission angles of sources with supersonic projected phase velocities (shown as broken lines) attain the limiting angles $\arcsin \left(\sin \alpha / M_{p}\right)$ and $\arccos \left(\cos \alpha / M_{p}\right)$ for $\sigma \rightarrow 0$ and $\sigma \rightarrow \infty$, respectively.

Supersonic oblique wave packets do not exhibit any surprising new emission patterns. We can find asymmetric Mach waves (similar to the results shown in figure 15) and oblique superdirective emission (similar to figure 13). Only the bidirective emission pattern (cf. figure 11c) that we have observed for $\alpha=0^{\circ}$ becomes now an asymmetric Mach wave emission which is oriented along the $x_{2}$-axis. In that sense, the supersonic bidirectivity from $\$ 3.2$ can also be regarded as an oblique Mach wave with respect to the $x_{2}$-axis which has a dominant emission angle of $\vartheta=90^{\circ}$.

\section{Origin of wave packet sources}

So far, we have only talked about generic sources $q(\boldsymbol{x}, t)$ with a Gaussian envelope, and one could argue that a source as defined in (3.1) does not exist in reality. Therefore, we would like to discuss, in this section, how our generic source fields $q(\boldsymbol{x}, t)$ relate to physical quantities.

If we assume that we have a pressure field $p(x, t)$ in the form of a wave packet with Gaussian envelope the tensor $\boldsymbol{T}_{i j}$ in Lighthill's analogy will only have diagonal entries. The Lighthill source term then reduces to $q(\boldsymbol{x}, t)=\partial^{2} p / \partial x_{i} \partial x_{i}$, i.e. the Laplacian of $p$. At first glance, this does not really seem to help us, since the Laplacian of a wave packet with Gaussian envelope is not a Gaussian wave packet anymore. In the spectral formulation of the Fraunhofer approximation (1.11), however, we find that the diagonal entries $\hat{\boldsymbol{T}}_{i i}$ are multiplied by $k_{i} k_{i}$, and because the Fourier modes of interest lie all on the acoustic circle $|\boldsymbol{k}|=k_{a}$, this factor (which in two dimensions is $k_{1}^{2}+k_{2}^{2}$ ) is exactly equal to $|\boldsymbol{k}|^{2}=k_{a}^{2}$. This means that a pressure field in the shape of a Gaussian wave packet emits sound exactly the same way as a generic Gaussian wave packet source $q$ (apart from the factor $k_{a}^{2}$ and the differences due to the Fraunhofer approximation). This is the case even though the Lighthill source term $\partial^{2} \boldsymbol{T}_{i j} / \partial x_{i} \partial x_{j}$ is not a Gaussian wave packet anymore. We have confirmed this fact numerically by computing the acoustic far field once for a pressure field $p$ according to Lighthill's analogy (1.3) and once for a generic source $q$ with corresponding Gaussian envelope. We find that the results are equivalent up to errors due to the Fraunhofer approximation.

The same reasoning works, of course, also for pure density fluctuations. In the context of Lighthill's analogy, however, the source field must be anisentropic if pressure and/or density fluctuations shall emit sound. Furthermore, the arguments made for pressure sources do not apply, in general, for the other terms of the Lighthill stress tensor (1.2), which involve velocities, e.g. $\rho u_{i} u_{j}$. Therefore, the example of the pure pressure source is not sufficient to establish the relevance of the present theory in the context of Lighthill's acoustic analogy.

A much better indication for its relevance is the observation that many source fields (near-field fluctuations) have actually the character of wave packets (e.g. Freund 2001). Stationary sources with Gaussian envelopes may arise, in particular, in the case of 
Mach wave radiation from instability modes in supersonic jets (Mitchell, Lele \& Moin 1997; Wu 2005). Moreover, the key ideas of the present theory can also be applied to non-Gaussian wave packets. As long as the contour lines of the sources in the wavenumber space are not too dissimilar from ellipses, the results presented here should hold approximately. At the same time, we should also stress that the present theory is limited to stationary envelopes. Wave packets sources which are convected and/or change their shape (e.g. Wu \& Huerre in press) are not covered by the present theory.

\section{Applications}

In this section, we would like to present two applications of the presented theory. These examples will show that the results derived in the previous sections are relevant to jet aeroacoustics. In particular, we will study two cases of sound emission from subsonic jets. The first example investigates the superdirective sound emission from low-Mach-number jets. The second example discusses the oblique emission of lowfrequency sound from highly subsonic jets with low Reynolds numbers.

\subsection{Superdirectivity in the experiment of Laufer \& Yen (1983)}

The analogy between pressure fields with Gaussian envelope and generic wave packet sources of the form (3.1) allows us to compare our results to the work of Crighton \& Huerre (1990) who studied superdirective emission patterns due to pressure boundary conditions. In contrast to our work, the boundary conditions are equivalent to pressure sources which are compact in the sideline direction $(\sigma \rightarrow \infty)$.

Crighton \& Huerre (1990) tried to explain a specific emission pattern observed experimentally by Laufer \& Yen (1983). Laufer \& Yen (1983) studied a low-Machnumber jet and found wave-packet-like structures in the jet which emit sound in a superdirective manner,

$$
\left\langle p^{2}\right\rangle \sim \exp \left(90 M_{p} \cos \varphi\right)
$$

where $\left\langle p^{2}\right\rangle$ is a measure for the sound intensity in the far field, i.e. $\mathrm{SPL}=\log \left\langle p^{2}\right\rangle+$ constant. These wave packets had a phase Mach number of $M_{p}=0.05$ and were only about two wavelengths long, i.e. $L \approx 2 \lambda_{0}$. With respect to the acoustic wavelength $\lambda_{a}=2 \pi a_{0} / \omega$ this wave packet was very short (approximately $0.1 \lambda_{a}$ ) and therefore 'acoustically compact'. Crighton \& Huerre (1990) asked the question 'How can such a small wave packet be superdirective?'

Based on the results established in $\S 3.3$, it is now relatively easy to answer this question. From (3.9) we see that the length scale $L$ is relevant for the difference $\triangle \mathrm{SPL}$ between the down- and upstream SPLs. However, it is only the aspect ratio $\sigma$ and the Mach number $M_{p}$ which determine whether the acoustic field has a superdirective topology (namely a single local maximum in the downstream direction).

From (6.1) we compute that the difference in the SPL between down- and upstream emissions is $\Delta \mathrm{SPL}=90 M_{p}-\left(-90 M_{p}\right)=9$. And according to (3.10) the relative curvature at $\varphi=0^{\circ}$ is $\kappa=1$ (which indicates the superdirective character). The two values $\triangle \mathrm{SPL}$ and $\kappa$ fully characterize the emission pattern, and we can use the formulas from $\S 3.3$ to determine the shape of the source field. From (3.9) and (3.11) we find that $\triangle \mathrm{SPL}=9$ is attained for

$$
\frac{L}{\lambda_{a}}=\sqrt{\frac{M_{p} \Delta \mathrm{SPL}}{8 \pi^{2}}} \approx 0.076
$$




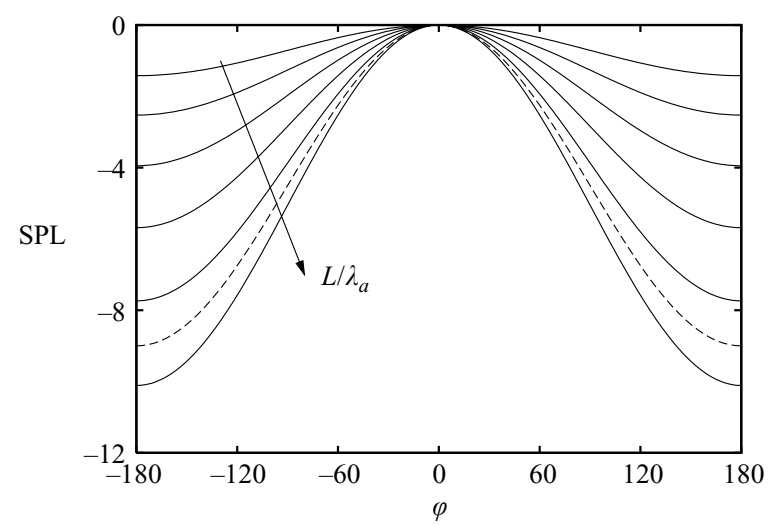

FIGURE 16. Numerically computed SPLs for $\sigma=1$ and $L / \lambda_{a}=0.03,0.04, \ldots, 0.08$. The dashed line indicates the result of Laufer \& Yen (1983). (The SPL curves are all shifted to a maximum value of 0 .)
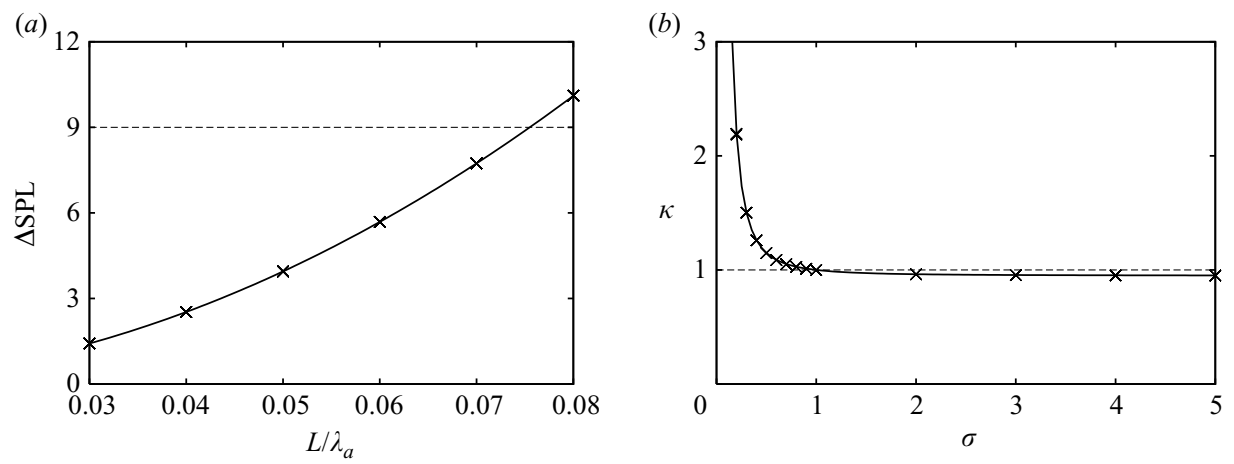

FIGURE 17. ( $a$ ) Difference between down- and upstream $\triangle$ SPL and $(b)$ curvature $\kappa$ for wave packets with $M_{p}=0.05, \sigma=0.1, \ldots, 10$ and $L / \lambda_{a}=0.3, \ldots, 0.8:-$, analytical predictions; $\times$, numerical results. Note that the solid lines (analytical predictions) collapse exactly in (a) for different $\sigma$ and in $(b)$ for different $L / \lambda_{a}$. The dashed lines indicate the values found by Laufer \& Yen (1983).

and that $\kappa=1$ corresponds to an aspect ratio of $\sigma=1$ (cf. figure 12). Given the difficulty of determining the width of a wave packet from experimental measurements (as already pointed out by Cooper \& Crighton 2000), we believe that our prediction for the width, $0.076 \lambda_{a}$, is reasonably close to the width of $0.1 \lambda_{a}$ reported by Laufer $\&$ Yen (1983). Further, result (6.2) illustrates again that acoustic compactness goes along well with superdirectivity. As a matter of fact, for the given Mach number superdirectivity would be obtained for all wave packets with aspect ratios $\sigma$ larger than $1 / \sqrt{21} \approx 0.22$.

We summarize that the emission pattern observed by Laufer \& Yen (1983) is consistent with a wave packet source with $M_{p}=0.05, \sigma \approx 1$ and $L \approx 0.076 \lambda_{a}$. To verify these results, we computed the acoustic emission from different acoustic sources by numerical integration of (1.3). Figures 16 and 17 compare these numerical results with our theoretical predictions and the findings of Laufer \& Yen (1983). In figure 17(b) we see that the relative curvature $\kappa$ changes only very little as $\sigma \rightarrow \infty$. Therefore, the 

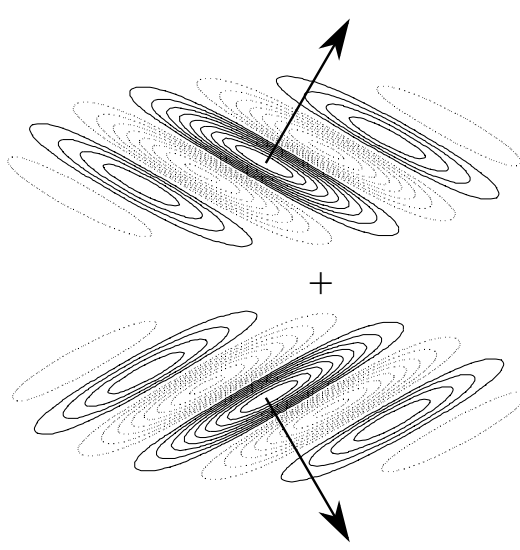

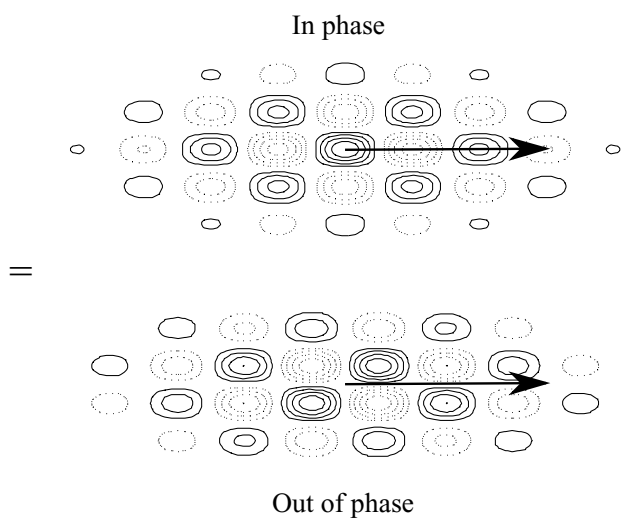

FIGURE 18. Construction of a pair of oblique wave packets with the wave packets in phase (top right) and out of phase (bottom right). The arrows indicate the direction of wave propagation.

results of Laufer \& Yen (1983) could also have been observed for aspect ratios much larger than unity.

With respect to the work of Crighton \& Huerre (1990), we would like to point out that their result for Gaussian wave packets is consistent with our findings. Namely the factor $M_{p} / \epsilon^{2}$ in the exponent of their equation (3.7) indicates that $\Delta \mathrm{SPL}=2 M_{p} / \epsilon^{2}$. According to our nomenclature their parameter $\epsilon$ corresponds to the ratio $\lambda_{0} /(2 \pi L)$, and therefore, they predicted exactly the same $\Delta$ SPL as we did in (3.9). It should be mentioned, however, that the main question raised by Crighton \& Huerre (1990) remains unanswered: the present theory does not give any indication why the experiments by Laufer \& Yen (1983) do not display any quadrupole character as suggested by Lighthill's analogy.

\subsection{Pairs of oblique wave packets}

An interesting consequence of our findings for oblique wave packets (cf. §4) is the directivity of the acoustic emission from pairs of oblique wave packets with $\boldsymbol{k}_{0}=\left(k_{0,1}, \pm k_{0,2}\right)$. The symmetry with respect to the $x_{1}$-axis leads to a standing wave in the $x_{2}$-direction. The waves propagate only in the $x_{1}$-direction with an apparent Mach number of $M_{p} / \cos \alpha$ (figure 18). Such a configuration is reminiscent of flow fields that we can find during the vortex pairing process in subsonic jets (see, for instance, figures 9, 12 and 13 in Sandham et al. 2006). Figure 19 shows two numerical experiments with pairs of subsonic oblique waves. Although the nominal phase Mach number is $M_{p}=0.4$ with a propagation angle $\alpha= \pm 60^{\circ}$, the waves in the source field appear to move only in the $x_{1}$-direction with phase speed $M_{p} / \cos \alpha=0.8$. If the pair is in phase (figure 19a) we obtain an emission pattern reminiscent of Mach waves with a local minimum at $\varphi=0^{\circ}$ and a dominant emission of about $30^{\circ}$. If the pair oscillates out of phase (figure 19b), the emission intensity in the downstream direction goes to zero because the sound waves emitted by the two wave packets cancel exactly along the $x_{1}$-axis. In that case, the Mach-wave-like emission pattern is even more pronounced.

These results show that a Mach-wave-like emission pattern can also be obtained for source fields with truly subsonic wave speed in the downstream direction. Unlike in the case shown in figure 15, neither the nominal Mach number nor its projections on to the $x_{1}$ - or $x_{2}$-axis are supersonic. As hinted above, source fields of this type can be found in laminar subsonic jet flows during the vortex pairing process. Depending 

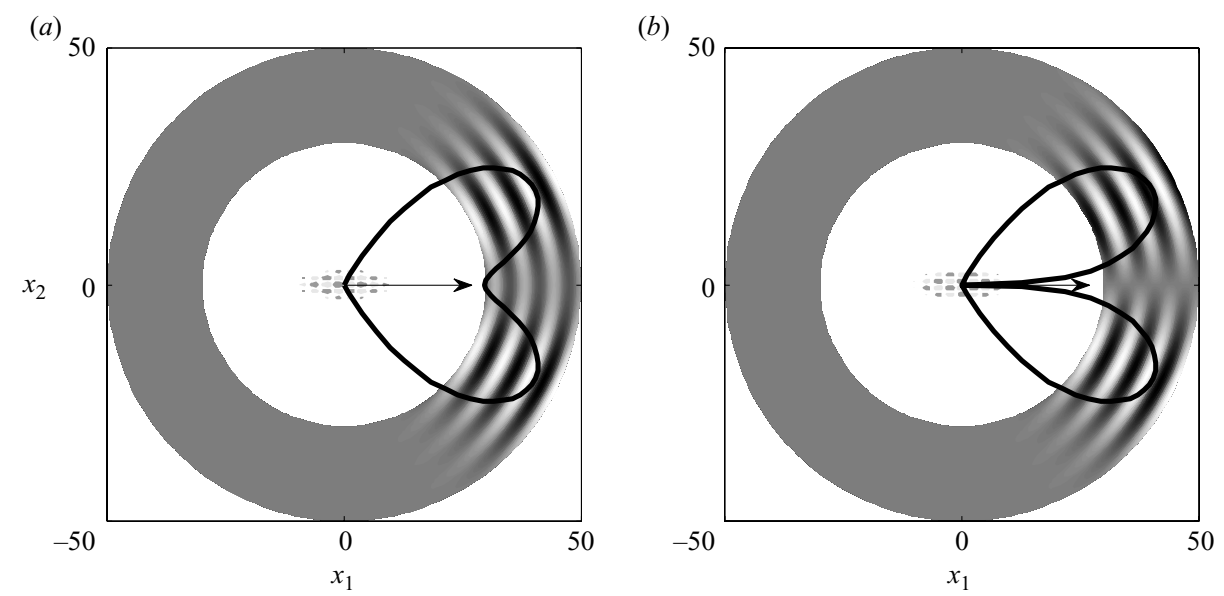

Figure 19. Pair of oblique wave packets with $M_{p}=0.4, \alpha= \pm 60^{\circ}, \sigma=3:(a)$ in phase, $(b)$ out of phase. (See the caption of figure $6 b$ for a more detailed explanation of these figures.)

on whether the vortices on opposite sides of the jet are staggered or not, the sound emission in the downstream direction $\varphi=0^{\circ}$ will be more or less silent. Anyway, the dominant direction of sound emission is oblique as also found in several numerical and physical experiments; see, for instance, figure 14 in Tam \& Morris (1980) or figure 10 in Freund (2001). The present theory offers a simple geometric explanation for this oblique directivity pattern.

\section{Summary and concluding remarks}

The present work describes a theory for the acoustic emission from wave packet sources to the far field. The foundation of this theory is an approximation to Lighthill's acoustic analogy for distant observers which is known as the Fraunhofer approximation. Because we transform this approximation to the frequencywavenumber domain the theory has a very illustrative character and uses simple geometric constructions.

The main concepts of the theory were introduced in $\S 2$ for one-dimensional problems. The criteria for uni- and bidirectional loudness, (2.4) and (2.5), are the main results of this section. With the extension to two spatial dimensions in $\S 3$ we introduced the aspect ratio $\sigma$ which turns out to be a key parameter for the acoustic emission pattern. In $\S 3.3$ we presented a unified theory for two-dimensional wave packets for which the wave propagation is aligned with one of the principal axes of the source field. Figure 12 - together with (3.9), (3.11) and (3.13) - is arguably the principal result of this paper. It illustrates the domains for bidirective, superdirective and Mach wave emissions as a function of the Mach number $M_{p}$ and the aspect ratio $\sigma$ and shows the corrected Mach angle. It should be emphasized at this point that we were able to find formulas which are surprisingly simple functions of the aspect ratio because $\sigma$ is a uniform parameter in our model; i.e. all (physical and spectral) amplitude contours of the source field are ellipses of the same aspect ratio. The two-dimensional theory has been generalized to oblique waves in $\S 4$. Equation (4.1) is the main result of this section. It describes the dominant angle of sound emission (with superdirective or Mach wave character) from wave packets with oblique waves. 
To relate our theory to actual acoustic source field, we showed in $\S 5$ that all results apply directly to pure pressure or density fluctuations in the shape of Gaussian wave packets. And we argued that wave packet sources have been observed in several acoustic source fields. As a first application of the presented theory, we used our results in $\S 6.1$ to explain experimental observations by Laufer \& Yen (1983). The study of this problem showed also that the results of Crighton \& Huerre (1990) for Gaussian envelopes are a special case of our theory for $\sigma \rightarrow \infty$. In a second application, we discussed the emission of pairs of oblique waves in $\S 6.2$ and suggested that some features of the acoustic emission pattern of subsonic jets can be explained by such pairs of oblique wave packets.

The proposed theory can serve as a tool for relating wave packet sources to an acoustic emission pattern. It stresses the fact that the the topology of the directivity pattern of a source field depends strongly on the phase Mach number and its aspect ratio.

\section{REFERENCES}

Avital, E. J. \& Sandham, N. D. 1997 A note on the structure of the acoustic field emitted by a wave packet. J. Sound. Vib. 204 (3), 533-539.

Bastin, F., LAFon, P. \& CANDEL, S. 1997 Computation of jet mixing noise due to coherent structures: the plane jet case. J. Fluid Mech. 335, 261-304.

Cooper, A. J. \& Crighton, D. G. 2000 Global modes and superdirective acoustic radiation in low-speed axisymmetric jets. Eur. J. Mech. B 19, 559-574.

Crighton, D. G. 1975 Basic principles of aerodynamic noise generation. Prog. Aerosp. Sci. 16 (1), 31-96.

Crighton, D. G. \& Huerre, P. 1990 Shear-layer pressure fluctuations and superdirective acoustic sources. J. Fluid Mech. 220, 355-368.

Freund, J. B. 2001 Noise sources in a low-Reynolds-number turbulent jet at Mach 0.9. J. Fluid Mech. 438, 277-305.

Goldstein, M. E. 2005 On identifying the true sources of aerodynamic sound. J. Fluid Mech. 526, 337-347.

Haj-Hariri, H. \& Akylas, T. R. 1986 Sound radiation by instability wavepackets in a boundary layer. Stud. Appl. Math. 75, 57-76.

Howe, M. S. 2003 Theory of Vortex Sound. Cambridge University Press.

Huerre, P. \& CRighton, D. G. 1983 Sound generation by instability waves in a low Mach number jet. In Eighth AIAA Aeroacoustics Conference, AIAA 83-0661, Atlanta, GA.

Kraichnan, R. H. 1953 The scattering of sound in a turbulent medium. J. Acoust. Soc. Am. 25, $1096-1104$.

Laufer, J. \& Yen, T.-C. 1983 Noise generation by a low-Mach-number jet. J. Fluid Mech. 134, 1-31.

LE Dizès, S. \& Millet, CH. 2007 Acoustic near field of a transonic instability wave packet. J. Fluid Mech. 577, 1-23.

LESSHAFF, L. 2006 Nonlinear global modes and sound generation in hot jets. PhD thesis, École Polytechnique, Paris.

Lighthill, J. 1952 On sound generated aerodynamically. Part I. General theory. Proc. R. Soc. Lond. A 211, 564-587.

Mitchell, B. E., Lele, S. K. \& Moin, P. 1997 Direct computation of Mach wave radiation in an axisymmetric supersonic jet. AIAA J. 35, 1574-1580.

Phillips, O. M. 1960 On the generation of sound by supersonic turbulent shear layers. J. Fluid Mech. 9, 1-28.

Sandham, N. D., Morfey, C. L. \& Hu, Z. W. 2006 Nonlinear mechanisms of sound generation in a perturbed parallel jet flow. J. Fluid Mech. 565, 1-23.

TAM, C. K. W. \& Burton, D. E. $1984 a$ Sound generated by instability waves of supersonic flows. Part 1. Two-dimensional mixing layers. J. Fluid Mech. 138, 249-271. 
TAM, C. K. W. \& Burton, D. E. $1984 b$ Sound generated by instability waves of supersonic flows. Part 2. Axisymmetric jets. J. Fluid Mech. 138, 273-295.

TAM, C. K. W. \& MorRIS, P. J. 1980 The radiation of sound by the instability waves of a compressible plane turbulent shear layer. J. Fluid Mech. 98 (2), 349-381.

Wu, X. 2005 Mach wave radiation of nonlinearly evolving supersonic instability modes in shear layers. J. Fluid Mech. 523, 121-159.

WU, X. \& HuERre, P. 2009 Low-frequency sound radiated by a nonlinearly modulated wavepackets of helical modes on a subsonic circular jet. J. Fluid Mech. 637, 173-211. 